\title{
Cromolyn Sodium Inhalant PA101
}

National Cancer Institute

\section{Source}

National Cancer Institute. Cromolyn Sodium Inhalant PA101. NCI Thesaurus. Code C123818.

A solution containing a high concentration of cromolyn sodium, with potential mast cell stabilizing, anti-tussive and anti-inflammatory activities. Upon inhalation of PA101 via a nebulizer, cromolyn blocks calcium ion influx into mast cells, thereby preventing the degranulation of mast cells in the lungs. This blocks the release of pro-inflammatory mediators from mast cells, such as histamine and slow-reacting substance of anaphylaxis (SRS-A), and prevents both bronchoconstriction and an inflammatory response. In indolent systemic mastocytosis (ISM), PA101 may be able to reduce the symptoms associated with this disease 